\title{
Evidenzorientierte Untersuchungs- und Behandlungsmethoden für Migränepatienten
}

\author{
Kerstin Lüdtke
}

\author{
Ungefähr 80\% aller Migräniker berichten, dass sie vor, während oder direkt im Anschluss an \\ ihre Kopfschmerzen unter Nackensymptomen leiden [4, 31]. Hat die Halswirbelsäule also \\ vielleicht einen Einfluss auf Migräne-Kopfschmerzen? Wäre dies tatsächlich der Fall, könnte \\ die Physiotherapie die Intensität und Frequenz der Schmerzattacken positiv beeinflussen.
}

Dass die Halswirbelsäule tatsächlich Kopfschmerzen hervorrufen, verstärken oder verlängern kann, ist relativ unumstritten: Es gibt einen Einfluss der Halswirbelsäule auf Kopfschmerzen. Die anatomische Grundlage hierfür ist die so genannte trigemino-zervikale Konvergenz, also die räumliche Nähe von Afferenzen aus dem trigeminalen und dem zervikalen System im Bereich des trigeminalen Nucleus. Die enge Nachbarschaft beider Systeme kann dazu führen, dass Informationen aus dem trigeminalen und dem zervikalen System vom Gehirn fehllokalisiert bzw. fehlinterpretiert werden $[6,7,11,12,20]$.

\section{Zentrale Mechanismen}

Tatsächlich scheint die Prävalenz von zervikogenen Kopfschmerzen in der Bevölkerung, also Kopfschmerzen, bei denen die Halswirbelsäule die Ursache ist, nur etwa 0,42,5\% auszumachen [21]. Migräne ist eine neurologische Erkrankung und mit ca. 16-20\% weitaus häufiger [40]. Die Fachwelt ist sich einig, dass v.a. zentrale Mechanismen - im Hirnstamm und/oder im Hypothalamus - eine entscheidende Rolle für das Auftreten der Attacken haben, während das trigeminale System für die Symptome verantwortlich ist $[1,38]$. Die früher gängige Meinung, dass es sich bei der Migräne um eine Erkrankung der Gefäße handelt, ist mittlerweile widerlegt, auch wenn die Gefäße am Krankheitsbild beteiligt sind [30].

Denkfehler Die hohe Prävalenz von Nackenschmerzen bei Migräne verleitet zu Verwechslungen der sekundären Kopfschmerzart „zervikogener Kopfschmerz“ mit der primären Kopfschmerzart „Migräne“. Dies geschieht allzu leicht dann, wenn nach den Kriterien von Sjaastad untersucht wird. Sjaastads Kriterien lassen bei der Diagnose zervikogener Kopfschmerzen auch assoziierte Symptome wie z. B. Übelkeit zu. Um solche Irrtümer zu vermeiden, greifen neurologische Kopfschmerzexperten zu den aktuellen
Diagnosekriterien der Internationalen Kopfschmerzgesellschaft (ICHD-III der International Headache Society, IHS).

Allodynie Warum leiden Migränepatienten so oft unter Nackenschmerzen? Manche Autoren verweisen auf eine generell erhöhte Empfindlichkeit von Migränikern. Gemessen wurde diese Hypersensitivität u.a. anhand von niedrigeren Druckschmerz-Schwellen [3]. Andere erkennen in diesem Phänomen eine Form der Allodynie, die wahrscheinlich nur unmittelbar vor und während der Attacken auftritt [20]. Dies würde bedeuten, dass vor oder während einer Schmerzattacke eine ansonsten indolente Spannung der Nackenmuskeln als schmerzhaft interpretiert wird. Zwischen den Attacken müssten Migränepatienten somit komplett „normal“, also nicht signifikant anders als Nicht-Migräniker sein. Einige Untersuchungen scheinen dies zu bestätigen [2, 26], während andere Studien von einer schlechteren Haltung [15], einer vermehrten Anzahl von Triggerpunkten [8, 14, 41] oder von Bewegungseinschränkungen $[8,41]$ im Vergleich mit NichtMigränikern berichten.

In einer bisher unveröffentlichten Literaturübersicht wurden ca. 25 Studien identifiziert, in denen eine Migränepopulation mittels eines körperlichen Tests untersucht wurde. Leider waren die Fallzahlen der meisten Studien sehr gering, die Diagnosekriterien uneinheitlich, die Verblindung der Untersucher nicht immer gewährleistet, so dass am Ende unklar bleibt, wie ein Patient mit Kopfschmerzen klinisch untersucht werden sollte.

\section{Tests für Kopfschmerzpatienten}

Auf Grund dieser Unsicherheit wurde im Jahre 2016 eine Initiative gestartet, deren Ziel ein internationaler Konsens für die körperliche Untersuchung von Kopfschmerz- 
patienten war. An der Delphi-Studie nahmen 17 Experten teil, bewerteten Tests und schlugen andere Untersuchungsmethoden vor. Schlussendlich einigte sich das Gremium auf elf Tests, die als Minimaluntersuchung für Kopfschmerzpatienten angesehen werden können [32] ( $\triangleright$ Abb. 1).

\section{Merke}

Minimaluntersuchung für Kopfschmerzpatienten Die Minimaluntersuchung umfasst 11 Tests:

1. aktives Bewegungsausmaß der HWS

2. Haltung, v.a. Kopftranslation nach ventral

3. BWS-Screening (aktive und passive Tests)

4. oberer zervikaler Quadrant, kombinierte Bewegung

5. kranio-zervikaler Flexionstest

6. manuelle Gelenktestung der oberen HWS

7. Reproduktion \& Resolution

8. Triggerpunkt-Palpation in Nacken und Gesicht

9. Überprüfung der Muskelkraft des Schultergürtels

10. passive physiologische Bewegungen der HWS

11. Flexions-Rotations-Test

Ziel der Kommission war die einheitliche Untersuchung auch unterschiedlicher Kopfschmerzarten. Ansonsten bestünde die Gefahr einer Fehldiagnose, oder ein Patient leidet unter mehr als einer Kopfschmerzart. In diesem Kontext berichten mehrere Autoren von koexistierenden Kopfschmerzarten - sei es eine Kombination aus Migräne und zervikogenem Kopfschmerz [39] oder aus Migräne und Spannungskopfschmerz $[9,43]$.

\section{PRAXIS-TIPP}

Bei der Befundung von Migränepatienten dürfen andere Tests nicht vernachlässigt werden, wenn die klinische Situation es erfordert.

Bei Kopfschmerz in Kombination mit Kiefersymptomen muss das Temporomandibular-Gelenk samt dazugehöriger Muskulatur mituntersucht werden [17]. Bei Schwindel ist die exakte neurologische/neurodynamische Untersuchung samt Gefäß- und LigamentTests für die obere Halswirbelsäule obligat.

\section{Haben Experten immer Recht?}

Der Delphi-Survey wiederspiegelt die Meinung internationaler Experten. Aber sind die 11 Tests nützlich für die praktische Untersuchung von Kopfschmerzpatienten? Dies wurde im Rahmen einer Studie hinterfragt. Im Fokus standen zunächst Migräniker.

Studienkriterien Für die Studie wurden 138 Patienten mit hochfrequent episodischer oder chronischer Migräne sowie 73 Probanden ohne Kopfschmerzen untersucht. Für den Zeitpunkt der Untersuchung entscheidend war, dass alle Probanden am Untersuchungs-Tag keine Kopfschmerzen hatten (chronische Migräne) und dass Personen mit episodischer Migräne überdies in den 48 Stunden vor der Untersuchung kopfschmerzfrei waren. Diese Kriterien waren wichtig, um nicht eine vermeintliche Allodynie vor oder während einer Migräneattacke zu untersuchen. Stattdessen galt es, Dysfunktionen zu identifizieren, die auch in der schmerzfreien Zeit vorhanden sind. Gäbe es solche Dysfunktionen, könnten diese mittels Physiotherapie positiv beeinflusst werden.

Studienergebnis Die Analyse der Untersuchungen offenbarte, dass sich Patienten mit Migräne tatsächlich in sechs der elf Tests - es handelt sich um folgende Untersuchungen: Triggerpunkt-Palpation in Nacken und Gesicht, Flexions-Rotations-Test, BWS-Screening, manuelle Gelenktestung der oberen HWS, kranio-zervikaler Flexions-Test sowie Reproduktion \& Resolution - signifikant von Personen ohne Kopfschmerzen unterscheiden [35]. Dabei zeigten rund 93\% der Migräniker mindestens drei verschiedene Dysfunktionen.

Angesichts der Testergebnisse ist eine Allodynie-Erklärung nicht ausreichend für die Begründung der typischen Nackenschmerzen bei Migräne. Stattdessen scheint es, dass die Migräne mit muskuloskeletalen Dysfunktionen korrespondiert - insbesondere in Form von Druckdolenzen und Triggerpunkten in den Nackenmuskeln sowie Bewegungseinschränkungen der oberen Halswirbelsäule.

Folgestudie Besonders die Ergebnisse der manuellen HWS-Testung waren überraschend: Es wurden derart viele positive Befunde apparent, dass in einer Folgestudie mit 40 zusätzlichen Teilnehmern nur der Respons auf die manuelle Untersuchung mit der normalen oszillierenden Technik sowie einer gehaltenen Technik ausgewertet wurde ( $\triangleright$ Abb. $\mathbf{1} \mathbf{g}$ und j). Es zeigte sich, dass $80 \%$ der Migränepatienten empfindlich auf lokalen Druck reagierten und dass dabei mehr als die Hälfte einen ausstrahlenden Schmerz in die Migräneschmerz-Region verspürte [34].

Trotz dessen bleibt bei dieser Untersuchung unklar, welche Struktur überhaupt schmerzempfindlich reagiert: Bei der Palpation von Gelenken kommt es auch zur Stimulation von Haut, Gefäßen, Muskeln, Bindegewebe, Faszien und Nervenfasern. Gleichzeitig ist ungewiss, warum Personen mit Migräne so deutliche Auffälligkeiten im Vergleich zu kopfschmerzfreien Personen aufweisen. Beeinflusst nun die Halswirbelsäule die Migräne; oder ist es die Migräne, die durch rezidivierende Muskelanspannungen die Empfindlichkeit und Beweglichkeit der oberen Halswirbelsäule verändert? 


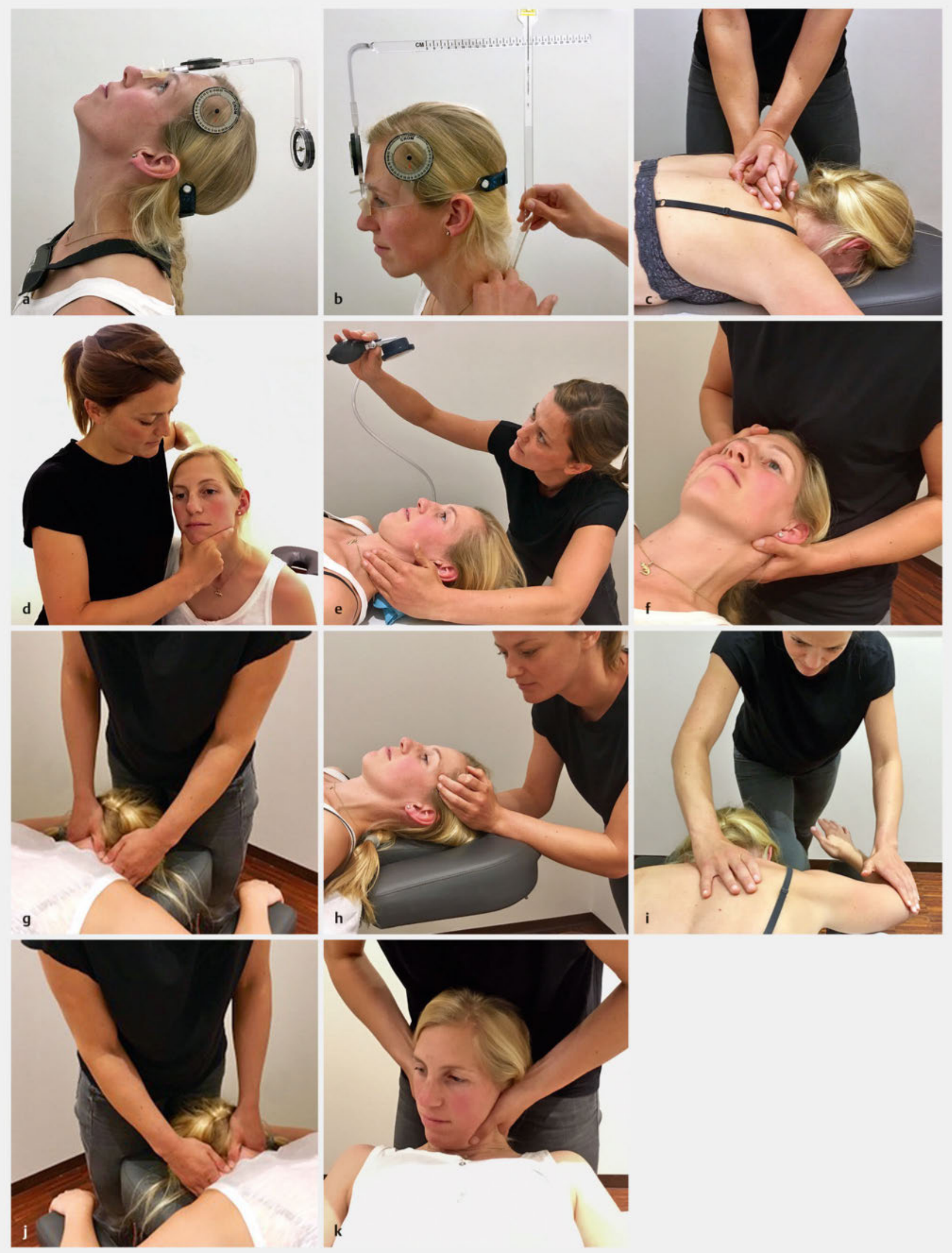

- Abb. 1 


\section{Evidenzbasierte Migräne-Therapie}

Der Einfluss einer HWS-Dysfunktion auf Migräne lässt sich also nur schwer abschätzen. Um diese Frage zu klären, kann die spezifische Therapie der in den elf Tests befundeten Dysfunktionen dienlich sein. Falls also eine HWS-Behandlung die Migräne-Symptomatik in jeglicher Form - Anzahl der Kopfschmerztage pro Monat, Intensität und Dauer der Kopfschmerz-Attacken - positiv beeinflusst, so würde dies die Relevanz der Halswirbelsäule für die Migräne bestätigen.

\section{Nicht-medikamentöse Migräne-Interventionen werden stiefmütterlich behandelt.}

Diesbezügliche randomisierte kontrollierte Studien (RCTs) existieren nicht. Die dünne Evidenzlage spiegelt sich in nationalen und internationalen Migräne-TherapieLeitlinien, die vorrangig Medikamente empfehlen. Bei den Arzneimitteln handelt es sich um Triptane für die Behandlung akuter Attacken; für chronische Migränepatienten werden Präventionsmedikamente gutgeheißen, um die Frequenz der Schmerz-Attacken zu reduzieren. Alternativen gibt es für die Betroffenen kaum - wenn überhaupt werden Entspannungstechniken, Biofeedback oder aerobes Training empfohlen [5].

\section{Die Rolle der Physiotherapie}

Eine aktuelle Analyse der Effektivität aktiver physiotherapeutischer Interventionen führt vor Augen, dass - mit Ausnahme einer einzigen Studie, die ein multimodales Programm untersuchte - bei Migränepatienten bislang lediglich die Effektivität aeroben Trainings evaluiert wurde [33]. Bei Spannungskopfschmerzen wurden physiotherapeutische Übungen, Manuelle Therapie sowie auch Triggerpunkt-Behandlungen mit etwas Erfolg eingesetzt $[13,19]$. Zervikogene Kopfschmerzen reagieren zuverlässig auf die Therapie von Triggerpunkten im Bereich der Nackenmuskulatur und auf die Manuelle Therapie der Halswirbelsäule [10, 22, 27].
De facto gibt es außer aerobem Training und Entspannungsverfahren keine evidenzbasierte physiotherapeutische Behandlung von Migräne. Eine wichtige Rolle, die auch in den Kompetenzbereich der Physiotherapie fällt, spielt die Edukation.

Migräne-Trigger Es scheint eine Reihe von Faktoren zu geben, welche die Migräne triggern. Insbesondere sind dies Stress, unregelmäßige Schlaf- und Essenszeiten sowie individuelle Faktoren. Im Allgemeinen sind potentielle Trigger-Faktoren bis dato noch nicht ausreichend untersucht. Möglich wäre, dass der Heißhunger auf Schokolade, Rotwein oder Käse bereits ein Teil der Migräne-Attacke ist und nicht - wie oftmals angenommen - diese Lebensmittel die Migräne auslösen. Dies würde erklären, warum bestimmte Lebensmittel manchmal zu Migräne führen und ansonsten völlig problemlos in größeren Mengen konsumiert werden können. Selbst der Einfluss von Wetterumschwüngen lässt sich wissenschaftlich nicht bestätigen, wenngleich Migräniker oft von diesem Phänomen berichten [25].

Aktivität statt Abhängigkeit Für die Physiotherapie ist es wichtig, da es sich bei der Migräne um eine chronische Erkrankung handelt, bei der Strategien des Selbstmanagements sinnvoller sind als jegliche Abhängigkeit von einem Therapeuten. Im Vordergrund sollen - entsprechend der Untersuchungsergebnisse aus den 11 Tests - aktive Behandlungselemente stehen. Wenn z. B. die Untersuchung der Brust- und Halswirbelsäule Steifigkeiten offenbart, können mobilisierende Übungen der entsprechenden Region Linderung verschaffen. Bei schlechter Skapula-Kontrolle oder bei auffälligem kraniozervikalem Flexionstest empfehlen sich stabilisierende Übungen für Schultergürtel und Halswirbelsäule. Dehnungen oder andere Weichteiltechniken helfen bei aktiven Triggerpunkten.

Physiotherapie wird keine Migräne heilen, aber die hohe Prävalenz von Zeichen und Symptomen im Bereich der Hals- und Brustwirbelsäule macht zumindest eine Reduktion der assoziierten Symptome denkbar. Wenn sich bewahrheitet, dass muskuloskeletale Dysfunktionen ein Mi-

\footnotetext{
- Abb. 111 Tests für Kopfschmerzpatienten. Quelle: K. Lüdtke (nachgestellte Situation).

a Aktives Bewegungsausmaß der HWS, gemessen mit einem CROM.

b Kopftranslation nach ventral, gemessen mit einem CROM.

c BWS-Screening.

d Oberer zervikaler Quadrant.

e Kranio-zervikaler Flexions-Test.

f Manuelle Gelenktestung der oberen HWS.

g Reproduktion \& Resolution (gehaltene Technik).

h Triggerpunkt-Palpation in Nacken und Gesicht.

i Überprüfung der Muskelkraft des Schultergürtels.

j Passive physiologische Bewegungen der HWS (oszillierende Technik).

k Flexions-Rotations-Test.
} 
gräne-Trigger sind, ist nach einer erfolgreichen Behandlung der Dysfunktion eine Reduktion der Kopfschmerztage pro Monat sehr wahrscheinlich.

\section{Autorinnnen/Autoren}

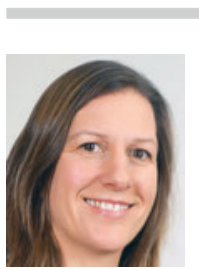

\section{Dr. Kerstin Lüdtke}

ist Physiotherapeutin und Postdoktorandin am Institut für systemische Neurowissenschaften am Universitätsklinikum Hamburg-Eppendorf. Ihr Forschungsschwerpunkt ist die Rolle der Physiotherapie bei Schmerzen und Kopfschmerzen.

\section{Korrespondenzadresse}

\section{Dr. Kerstin Lüdtke}

Physiotherapeutin, Post-Doc

Institut für systemische Neurowissenschaften

Universitätsklinikum Hamburg-Eppendorf

20246 Hamburg

k.luedtke@uke.de

\section{Literatur}

[1] Aderjan D, Stankewitz A, May A. Neuronal mechanisms during repetitive trigemino-nociceptive stimulation in migraine patients. Pain 2010; 151: 97-103. doi:10.1016/j. pain.2010.06.024

[2] Amiri M, Jull G, Bullock-Saxton J, et al. Cervical musculoskeletal impairment in frequent intermittent headache. Part 2: subjects with concurrent headache types. Cephalalgia 2007; 27 : 891-898. doi:10.1111/j.1468-2982.2007.01346.x

[3] Andersen S, Petersen MW, Svendsen AS et al. Pressure pain thresholds assessed over temporalis, masseter, and frontalis muscles in healthy individuals, patients with tension-type headache, and those with migraine-a systematic review. Pain 2015; 156: 1409-1423. doi:10.1097/j.pain.0000000000 000219

[4] Ashina S, Bendtsen L, Lyngberg AC et al. Prevalence of neck pain in migraine and tension-type headache: A population study. Cephalalgia 2015; 35: 211-219. doi:10.1177| 0333102414535110

[5] Arbeitsgemeinschaft der Wissenschaftlichen Medizinischen Fachgesellschaften e.V. (AWMF). 2012. Im Internet: http:// www.awmf.org/leitlinien/aktuelle-leitlinien/II-liste/deutschemigraene-und-kopfschmerzgesellschaft.html; Stand: 05.01.2018

[6] Bartsch T, Goadsby PJ. Increased responses in trigeminocervical nociceptive neurons to cervical input after stimulation of the dura mater. Brain 2003; 126: 1801-1813. doi:10.1093/ brain/awg190

[7] Bartsch T, Goadsby PJ. The trigeminocervical complex and migraine: current concepts and synthesis. Curr Pain Headache Rep 2003; 7: 371-376

[8] Bevilaqua-Grossi D, Pegoretti KS, Goncalves MC et al. Cervical mobility in women with migraine. Headache 2009; 49: 726731. doi:10.1111/j.1526-4610.2008.01233.x

[9] Blumenfeld A, Schim J, Brower J. Pure tension-type headache versus tension-type headache in the migraineur. Curr Pain Headache Rep 2010; 14: 465-469. doi:10.1007/s11916-0100147-1
[10] Bodes-Pardo G, Pecos-Martín D, Gallego-Izquierdo T et al. Manual treatment for cervicogenic headache and active trigger point in the sternocleidomastoid muscle: a pilot randomized clinical trial. J Manipulative Physiol Ther 2013; 36: 403411. doi:10.1016/j.jmpt.2013.05.022

[11] Bogduk N. The neck and headaches. Neurol Clin 2014; 32: 471-487. doi:10.1016/j.ncl.2013.11.005

[12] Bogduk N. The anatomical basis for cervicogenic headache. J Manipulative Physiol Ther 1992; 15: 67-70

[13] Castien RF, van der Windt DAWM, Grooten A et al. Effectiveness of manual therapy for chronic tension-type headache: a pragmatic, randomised, clinical trial. Cephalalgia 2011; 31: 133-143. doi:10.1177/0333102410377362

[14] Fernández-de-Las-Peñas C, Cuadrado ML, Pareja, JA. Myofascial trigger points, neck mobility and forward head posture in unilateral migraine. Cephalalgia 2006; 26: 1061-1070. doi:10.1111/j.1468-2982.2006.01162.x

[15] Ferreira MC, Bevilaqua-Grossi D, Dach FE et al. Body posture changes in women with migraine with or without temporomandibular disorders. Braz J Phys Ther 2014; 18: 19-29

[16] Fletcher JP, Bandy WD. Intrarater reliability of CROM measurement of cervical spine active range of motion in persons with and without neck pain. J Orthop Sports Phys Ther 2008; 38 : 640-645. doi:10.2519/jospt.2008.2680

[17] Florencio LL, de Oliveira AS, Carvalho GF et al. Association Between Severity of Temporomandibular Disorders and the Frequency of Headache Attacks in Women With Migraine: A Cross-Sectional Study. J Manipulative Physiol Ther 2017; 40: 250-254. doi:10.1016/j.jmpt.2017.02.006

[18] Garrett TR, Youdas JW, Madson TJ. Reliability of measuring forward head posture in a clinical setting. J Orthop Sports Phys Ther 1993; 17: 155-160. doi:10.2519/jospt.1993.17.3.155

[19] Ghanbari A, Rahimijaberi A, Mohamadi M et al. The effect of trigger point management by positional release therapy on tension type headache. NeuroRehabilitation 2012; 30: 333339. doi:10.3233/NRE-2012-0764

[20] Goadsby P]. Migraine, allodynia, sensitisation and all of that. Eur. Neurol. 2005; 53 (Suppl 1): 10-16. doi:10.1159/ 000085060

[21] Haldeman S, Dagenais S. Cervicogenic headaches: a critical review. Spine J 2001; 1: 31-46. doi:10.1016/S1529-9430(01) 00024-9

[22] Hall T, Chan HT, Christensen L et al. Efficacy of a C1-C2 selfsustained natural apophyseal glide (SNAG) in the management of cervicogenic headache. J Orthop Sports Phys Ther 2007; 37: 100-107. doi:10.2519/jospt.2007.2379

[23] Hall T, Robinson $\mathrm{K}$. The flexion-rotation test and active cervical mobility - a comparative measurement study in cervicogenic headache. Man Ther 2004; 9: 197-202. doi:10.1016/j. math.2004.04.004

[24] Headache Classification Committee of the International Headache Society (IHS). The International Classification of Headache Disorders, 3rd edition (beta version). Cephalalgia 2013; 33: 629-808. doi:10.1177/0333102413485658

[25] Hoffmann J, Schirra T, Lo $\mathrm{H}$ et al. The influence of weather on migraine - are migraine attacks predictable? Ann Clin Transl Neurol 2015; 2: 22-28. doi:10.1002/acn3.139

[26] Jull G, Amiri M, Bullock-Saxton J et al. Cervical musculoskeletal impairment in frequent intermittent headache. Part 1: Subjects with single headaches. Cephalalgia 2007; 27: 793-802. doi:10.1111/j.1468-2982.2007.01345.x 
[27] Jull G, Trott P, Potter $\mathrm{H}$ et al. A randomized controlled trial of exercise and manipulative therapy for cervicogenic headache. Spine 2002; 27: 1835-1843

[28] Jull G, O'Leary SP, Falla DL. Clinical assessment of the deep cervical flexor muscles: the craniocervical flexion test. J Manipulative Physiol Ther 2008; 31: 525-533. doi:10.1016/j. jmpt.2008.08.003

[29] Kendall FP, McCreary EK, Provance PG et al. Muscles: Testing and Function, with Posture and Pain. 5th ed. Baltimore: Lippincott Williams \& Wilki; 2010

[30] Kurth T, Dodick DW. Migraine and the vascular system: much has been learned but still a long way to go. Cephalalgia 2015; 35: 83-84. doi:10.1177/0333102414566050

[31] Lampl C, Rudolph M, Deligianni Cl et al. Neck pain in episodic migraine: premonitory symptom or part of the attack? J Headache Pain 2015; 16: 566. doi:10.1186/s10194-0150566-9

[32] Luedtke K, Boissonnault W, Caspersen N et al. International consensus on the most useful physical examination tests used by physiotherapists for patients with headache: A Delphi study. Manual Therapy 2016; 23: 17-24 [in Revison]. doi:10.1016/j.math.2016.02.010

[33] Luedtke K, Allers A, Schulte LH et al. Efficacy of interventions used by physiotherapists for patients with headache and migraine-systematic review and meta-analysis. Cephalalgia 2015; 36: 474-492. doi:10.1177/0333102415597889

[34] Luedtke K, May A. Stratifying migraine patients based on dynamic pain provocation over the upper cervical spine. Journal of headache and Pain 2017 [submitted]

[35] Luedtke K, Starke W, May A. Musculoskeletal dysfunction in migraine patients. Cephalalgia 2017; 333102417716934. doi:10.1177/0333102417716934

[36] Maitland GD. Vertebral Manipulation. 2013. 4th ed. LondonBoston: Butterworth-Heinemann; 2013

[37] Moore MK. Upper crossed syndrome and its relationship to cervicogenic headache. J Manipulative Physiol Ther 2004; 27 : 414-420. doi:10.1016/j.jmpt.2004.05.007
[38] Schulte LH, May A. The migraine generator revisited: continuous scanning of the migraine cycle over 30 days and three spontaneous attacks. Brain 2016; 139: 1987-1993. doi:10.1093/brain/aww097

[39] Sjaastad O, Fredriksen T, Pareja JA et al. Coexistence of cervicogenic headache and migraine without aura (?). Funct. Neurol. 1999; 14: 209-218

[40] Smitherman TA, Burch R, Sheikh $\mathrm{H}$ et al. The prevalence, impact, and treatment of migraine and severe headaches in the United States: a review of statistics from national surveillance studies. Headache 2013; 53: 427-436. doi:10.1111/ head. 12074

[41] Tali D, Menahem I, Vered E et al. Upper cervical mobility, posture and myofascial trigger points in subjects with episodic migraine: Case-control study. J Bodyw Mov Ther 2014; 18: 569-575. doi:10.1016/j.jbmt.2014.01.006

[42] Travell JG, Simons, DG. Myofascial Pain and Dysfunction. The Trigger Point Manual. Vol. 1, 2nd edition. Baltimore: Lippincott Williams \& Wilki; 1998

[43] Ulrich V, Russell MB, Jensen R et al. A comparison of tensiontype headache in migraineurs and in non-migraineurs: a population-based study. Pain 1996; 67: 501-506

[44] Watson DH, Drummond PD. Head pain referral during examination of the neck in migraine and tension-type headache. Headache 2012; 52, 1226-1235. doi:10.1111/j.15264610.2012.02169.x

Bibliografie

DOI https://doi.org/10.1055/s-0044-100238

Der Schmerzpatient 2018; 1: 76-81

(c) Georg Thieme Verlag KG Stuttgart · New York ISSN 2512-6210 